Maria A. Rocca, MD

Martina Absinta, MD

Maria Pia Amato, MD

Lucia Moiola, MD

Angelo Ghezzi, MD

Pierangelo Veggiotti, MD

Ruggero Capra, MD

Emilio Portaccio, MD

Agnese Fiorino, PhD

Lorena Pippolo, MD

Maria Carmela Pera, MD

Mark A. Horsfield, PhD

Andrea Falini, MD

Giancarlo Comi, MD

Massimo Filippi, MD

Correspondence to

Prof. Filippi:

m.filippi@hsr.it

Editorial, page 1296

Supplemental data at Neurology.org

\title{
Posterior brain damage and cognitive impairment in pediatric multiple sclerosis
}

\section{ABSTRACT}

Objective: We combined structural and functional MRI to better understand the mechanisms responsible for cognitive impairment in pediatric patients with multiple sclerosis (MS).

Methods: Brain dual-echo, diffusion tensor, 3D T1-weighted, and resting-state (RS) fMRI scans were acquired from 35 consecutive pediatric patients with MS and 16 sex- and age-matched healthy controls. Patients with abnormalities in $\geq 2$ neuropsychological tests were classified as cognitively impaired. The regional distribution of white matter (WM) and gray matter (GM) damage was assessed using voxel-wise analyses. Default mode network (DMN) RS functional connectivity (FC) was also measured.

Results: Sixteen patients (45\%) were classified as cognitively impaired. Compared to cognitively preserved (CP) patients, cognitively impaired patients with MS had higher occurrence of T2 lesions as well as more severe damage to the WM and GM, as measured by atrophy and diffusivity abnormalities, in the posterior regions of the parietal lobes close to the midline (precuneus, posterior cingulum, and corpus callosum). Compared to the other study groups, they also showed reduced RS FC of the precuneus, whereas CP patients experienced an increased RS FC of the anterior cingulate cortex. A multivariable model identified diffusivity abnormalities of the cingulum and corpus callosum and RS FC of the precuneus as the covariates more strongly associated with cognitive impairment (C-index $=0.99)$.

Conclusions: In pediatric patients with MS, cognitive dysfunction is associated with structural and functional abnormalities of the posterior core regions of the DMN. WM structural abnormalities co-occurring at this level are likely to be the substrate of such modifications. Neurology ${ }^{\circledR}$ 2014;82:1314-1321

\section{GLOSSARY}

ACC $=$ anterior cingulate cortex; BNBC $=$ Brief Neuropsychological Battery for Children; $\mathbf{C C}=$ corpus callosum; $\mathbf{C P}=$ cognitively preserved; $\mathbf{D M N}=$ default mode network; $\mathbf{D T}=$ diffusion tensor; EDSS = Expanded Disability Status Scale; $\mathbf{E P I}=$ echoplanar imaging; FA = fractional anisotropy; $\mathbf{F C}=$ functional connectivity; FFE = fast field echo; $\mathbf{G M}=$ gray matter; HC = healthy controls; ICV = intracranial volume; ILF = inferior longitudinal fasciculus; $\mathbf{L}=$ left; $\mathbf{L P M}=$ lesion probability map; $\mathbf{L V}=$ lesion volume; $\mathbf{M D}=$ mean diffusivity; $\mathbf{M N I}=$ Montreal Neurological Institute; $\mathbf{M S}=$ multiple sclerosis; $\mathbf{M T G}=$ middle temporal gyrus; $\mathbf{R}$ = right; $\mathbf{R D}=$ radial diffusivity; $\mathbf{R F}$ = random forest; $\mathbf{R R M S}=$ relapsing-remitting multiple sclerosis; $\mathbf{R S}=$ resting state; $\mathbf{S E}=$ spin echo; $\mathbf{S L F}=$ superior longitudinal fasciculus; $\mathbf{S P A R T}=$ Spatial Recall Test; $\mathbf{S R T}=$ Selective Reminding Test; TBSS $=$ Tract-Based Spatial Statistics; TMT = Trail Making Test; $\mathbf{W M ~}=$ white matter .

Up to $10 \%$ of patients with multiple sclerosis (MS) experience their first attack during childhood. ${ }^{1}$ The transition to a secondary progressive course and accumulation of permanent disability is more gradual and takes longer in these patients than in those with adult-onset MS. ${ }^{2}$ The relatively limited disease-related damage ${ }^{3}$ with preservation of structural ${ }^{4,5}$ and functional ${ }^{6}$ integrity of the brain gray matter (GM) has been considered among the factors responsible for the course of pediatric MS.

A large proportion of pediatric patients with MS experience cognitive deficits, with a prominent involvement of linguistic abilities in addition to memory, attention, and executive

From the Neuroimaging Research Unit (M.A.R., M.A., M.F.), Department of Neurology, Institute of Experimental Neurology, Division of Neuroscience (M.A.R., M.A., L.M., A. Fiorino, G.C., M.F.), and Department of Neurology (M.P.A., E.P.), University of Florence; the Multiple Sclerosis Center (A.G., L.P.), Ospedale di Gallarate; Fondazione "Istituto Neurologico Casimiro Mondino" (P.V., M.C.P.), Pavia; Multiple Sclerosis Center (R.C.), Spedali Civili of Brescia, Italy; Department of Cardiovascular Sciences (M.A.H.), University of Leicester, UK; and the Department of Neuroradiology (A. Falini), San Raffaele Scientific Institute, Vita-Salute San Raffaele University, Milan, Italy.

Go to Neurology.org for full disclosures. Funding information and disclosures deemed relevant by the authors, if any, are provided at the end of the article. 
functions. ${ }^{7}$ The factors associated with cognitive impairment remain largely unexplored. More severe atrophy of the thalamus and corpus callosum (CC) has been found in cognitively impaired than in cognitively preserved (CP) pediatric patients with $\mathrm{MS}^{8}{ }^{8}$ Executive deficits have been correlated with atrophy of the thalamus and frontal lobes, ${ }^{9}$ while reduced cognitive speed $^{10}$ and math performance ${ }^{11}$ were correlated with CC damage.

To improve our understanding of the mechanisms responsible for the presence and severity of cognitive impairment in pediatric patients with MS, we applied a voxel-wise analysis of advanced structural MRI techniques to determine the patterns of regional involvement of the white matter (WM) and GM according to their cognitive profile. We also quantified resting-state (RS) functional connectivity (FC) of the default mode network (DMN), since this is the most robust network identified by RS studies of the human brain, and its disruption has been associated with cognitive deficits in elderly individuals ${ }^{12}$ and adult patients with MS. ${ }^{13-15}$

METHODS Patients. We studied 35 consecutive righthanded pediatric patients with relapsing-remitting MS (RRMS) referred to specialized MS centers and 16 sex- and age-matched right-handed healthy controls (HC) (table 1). Patients with acute disseminated encephalomyelitis were excluded according to published operational criteria. ${ }^{16}$ Patients had to be relapseand steroid-free for at least 3 months. Exclusion criteria were concomitant therapy with antidepressants, psychoactive drugs, or a history of major medical, neurologic, or psychiatric disorders.
Standard protocol approvals, registrations, and patient consents. Ethics committee approval. Approval was received from the local ethical standards committee on human experimentation, and written informed consent was obtained from all subjects' parents prior to study enrollment.

Clinical and neuropsychological evaluation. All patients underwent a neurologic examination with rating of the Expanded Disability Status Scale (EDSS), and a neuropsychological assessment using the Brief Neuropsychological Battery for Children (BNBC), which has been standardized and validated for Italian pediatric patients with MS. ${ }^{17}$ Both assessments were performed within 3 days of the MRI study by an experienced observer blinded to the clinical and MRI results. The BNBC includes the following: (1) global cognitive functioning with IQ; (2) verbal learning and delayed recall with the Selective Reminding Test (SRT, SRT-Delayed); (3) visuospatial learning and delayed recall with the Spatial Recall Test (SPART, SPART-Delayed); (4) sustained attention and concentration with the Symbol Digit Modalities Test and the Trail Making Test (TMT-A and TMT-B); (5) abstract reasoning through the Modified Card Sorting Test; (6) expressive language through a Semantic and Phonemic verbal fluency test and an Oral Denomination test; and (7) receptive language using the Token Test, the Indication of Pictures from the Neuropsychological Examination for Aphasia, and the Phrase Comprehension test from the Battery for the Analysis of Aphasic Deficits. The 5th or 95th percentile of the corrected scores of the normative data were used as the cutoff for determining failure at a given test. Patients with an abnormal performance in $\geq 2$ tests were classified as cognitively impaired. ${ }^{17}$

MRI acquisition. The e-Methods on the Neurology ${ }^{\circledR}$ Web site at Neurology.org provide a detailed description of the MRI acquisition and analysis protocol. Using a 3.0-T scanner, the following brain sequences were acquired, in the following order: T2*-weighted single-shot echoplanar imaging (EPI) sequence for RS fMRI; dual-echo turbo spin echo (SE); 3D T1-weighted fast field echo (FFE); and pulsed-gradient SE EPI with diffusion gradients applied in 35 noncollinear directions. The total acquisition duration was around 35 minutes.

MRI postprocessing and statistical analysis. MRI analyses were performed by an experienced observer blinded to the clinical and neuropsychological results. T2 lesion volumes (LV) were

Table 1 Main demographic, clinical, and conventional MRI characteristics of the subjects enrolled in the study

\begin{tabular}{|c|c|c|c|c|}
\hline & \multirow[b]{2}{*}{ Pediatric healthy controls } & \multicolumn{3}{|c|}{ Pediatric patients with RRMS } \\
\hline & & All & Cognitively preserved & Cognitively impaired \\
\hline Number of subjects & 16 & 35 & 19 & 16 \\
\hline Girls/boys & $9 / 7$ & $21 / 14$ & $13 / 6$ & $9 / 7$ \\
\hline Mean (range) age, y & $14.3(8-18)$ & $15.3(7-18)$ & $15.4(11-18)$ & $15.2(7-18)$ \\
\hline Mean (SD) education, y & $9.2(4.0)$ & $8.9(2.3)$ & 8.9 (2.3) & $8.9(2.3)$ \\
\hline Median (range) EDSS score & - & $1.5(0-3.5)$ & $1.5(0-3.0)$ & $1.5(0-3.5)$ \\
\hline Mean (range) disease duration, $y$ & - & $2.1(0.2-8.1)$ & $1.7(0.3-5.1)$ & $2.8(0.2-8.1)$ \\
\hline $\begin{array}{l}\text { Therapy: none/IFN- } \beta 1 \mathrm{a} / \text { glatiramer } \\
\text { acetate/natalizumab/mitoxantrone }\end{array}$ & - & $6 / 19 / 4 / 5 / 1$ & $4 / 10 / 3 / 2 / 0$ & $2 / 9 / 1 / 3 / 1$ \\
\hline Mean (SD) T2 LV, mL & - & $5.4(6.9)$ & $3.5(1.8)$ & $7.7(9.8)$ \\
\hline Mean (SD) T1 LV, mL & - & 3.4 (5.2) & $1.9(5.2)$ & $5.2(7.3)$ \\
\hline Mean (SD) ICV, mL & 1,450 (108) & $1,392(113)$ & $1,379(113)$ & $1,403(115)$ \\
\hline
\end{tabular}

Abbreviations: EDSS $=$ Expanded Disability Status Scale; ICV = intracranial volume; IFN- $\beta=$ interferon- $\beta$; LV $=$ lesion volume; RRMS = relapsing-remitting multiple sclerosis. 
quantified using a local thresholding segmentation technique (Jim 5, Xinapse Systems Ltd., Northants, UK). T1 hypointense lesions were identified and segmented on the 3D FFE images, which were previously coregistered and resliced to match the T2-weighted scans.

Analysis of structural regional WM and GM damage. The e-Methods provides a comprehensive methodologic description of the analysis used to assess structural abnormalities of the GM and WM.

Lesion probability maps (LPMs) were produced and compared between cognitively impaired and CP patients (2-sample $t$ test, SPM8).

Voxel-based morphometry was performed using SPM8 software. Between-group comparisons of GM and WM maps were assessed using analyses of covariance, including age, sex, and intracranial volume (ICV) as covariates. A conjunction analysis was run to identify atrophied areas in cognitively impaired patients vs $\mathrm{CP}$ patients and HC. A linear regression analysis was used to investigate the correlations between areas of regional atrophy associated with cognitive impairment vs clinical variables, neuropsychological scores, and other MRI variables (T2 and T1 LV).

Tract-Based Spatial Statistics (TBSS) analysis ${ }^{18}$ was used for voxel-wise analysis of whole brain WM diffusion tensor (DT) MRI measures. Voxel-wise differences in fractional anisotropy (FA), mean diffusivity (MD), axial diffusivity, and radial diffusivity (RD) values between pediatric patients and controls as well as between cognitively impaired and CP patients were tested using a permutation-based inference for nonparametric statistical thresholding ("randomize" program, FSL) ${ }^{19}$ and 2-sample $t$ tests, adjusting for age and T2 lesion maps. A nonparametric statistical thresholding was also used to assess the correlations between DT MRI metrics identified by the previous analysis and clinical and neuropsychological variables.

DMN RS FC analysis. A comprehensive methodologic description of the fMRI analyses is provided in the e-Methods. RS fMRI data were preprocessed using SPM8. An independent component analysis was used to decompose RS fMRI data into spatially independent maps and time courses (GIFT software). ${ }^{20}$ The DMN was identified through a voxel-wise correlation with the DMN template supplied in GIFT. ${ }^{21}$ Between-group comparisons of RS FC were tested using factorial models, which were explicitly masked with the binarized DMN mask extracted from each one-sample $t$ test using Marsbar. ${ }^{22}$ Correlations between DMN RS FC abnormalities vs clinical variables, neuropsychological scores, and other MRI variables were assessed using linear regression models.

Multimodal analysis. To integrate structural and RS functional MRI measures, multimodal prediction models were built to define which of the measures significantly different between groups predicted cognitive impairment in patients. To do this, a random forest (RF) approach (see e-Methods) ${ }^{23}$ was used to rank the MRI variables according to their importance (ability to predict the outcome). Thereafter, for each outcome, a multivariable logistic predictive model was built, using only the most important variables detected by the RF analyses. The final predictive model was chosen according to the minimum Akaike Information Criterion. The discriminatory power was assessed by computing C-statistics.

RESULTS Clinical, neuropsychological, and conventional MRI assessment. Age $(p=0.24)$ and $\operatorname{sex}(p=0.32)$ did not differ between pediatric $\mathrm{HC}$ and patients with MS. Sixteen pediatric patients with MS (45\%) were classified as cognitively impaired. Age $(p=0.2)$, disease duration $(p=0.1)$, EDSS score $(p=0.8)$, T2 LV $(p=0.1)$, T1 LV $(p=0.09)$, and ICV $(p=0.5)$ did not differ between cognitively impaired and CP patients with MS. The median number of abnormal neuropsychological tests was 0 (range $=0-1$, where 0 reflects no impairment) in $\mathrm{CP}$ patients and 3 (range = 2-9) in cognitively impaired patients. Table e-1 summarizes the performance on individual neuropsychological tests by pediatric patients with MS. Ten (31\%) patients showed a low $\mathrm{IQ}^{24}$ (range 70-90), including 5 (28\%) $\mathrm{CP}$ and $5(36 \%)$ cognitively impaired patients. The cognitive domains most frequently involved were spatial and verbal memory (17.6\% of all pediatric patients with MS, $1 \%$ of CP, $37.8 \%$ of cognitively impaired patients with MS), language abilities ( $11.5 \%$ of all pediatric patients with MS, $6.6 \%$ of CP, $17.8 \%$ of cognitively impaired patients), and attention ( $8 \%$ of all pediatric patients with MS, $1 \%$ of CP, $15.3 \%$ of cognitively impaired patients).

WM LPMs. Compared to CP patients, cognitively impaired patients had an increased probability of harboring lesions in the right $(\mathrm{R})$ thalamus, middle and posterior (close to the $\mathrm{R}$ precuneus) $\mathrm{CC}$, and bilateral parieto-occipital WM $(p<0.001$, uncorrected $)$ (figure 1).

GM atrophy. Table e-2 summarizes regions showing significant differences of GM volume between patients with MS and $\mathrm{HC}$ as well as between patient subgroups ( $p<0.001$, uncorrected). Compared to $\mathrm{CP}$ patients, cognitively impaired patients had atrophy of the R precuneus and left (L) middle temporal gyrus (MTG) (figure 1). No GM area was more atrophied in CP vs cognitively impaired patients. The conjunction analysis showed that, compared to $\mathrm{HC}$ and CP patients, cognitively impaired patients had atrophy of the R precuneus (Montreal Neurological Institute [MNI] coordinates: 11, $-59,36$ ) (figure 1).

WM atrophy. Table e-3 summarizes areas showing significant differences of WM volume between groups ( $p<0.001$, uncorrected). Compared to HC, pediatric patients with MS had atrophy of the $\mathrm{R}$ superior longitudinal fasciculus (SLF) and $\mathrm{L}$ inferior longitudinal fasciculus (ILF). Compared to HC, cognitively impaired patients had WM atrophy of the splenium of the CC, R SLF, WM close to L precuneus, and L ILF. No area of WM atrophy was identified in CP patients vs HC. Compared to CP patients, cognitively impaired patients had WM atrophy of the splenium of the CC, posterior cingulum, L parahippocampus, WM close to the precuneus, bilaterally, and bilateral SLF (figure 1). No area of WM atrophy was detected in $\mathrm{CP}$ vs cognitively impaired patients. The conjunction analysis showed that compared to HC and CP patients, cognitively impaired patients had WM atrophy 
Figure 1 Structural regional damage in pediatric multiple sclerosis

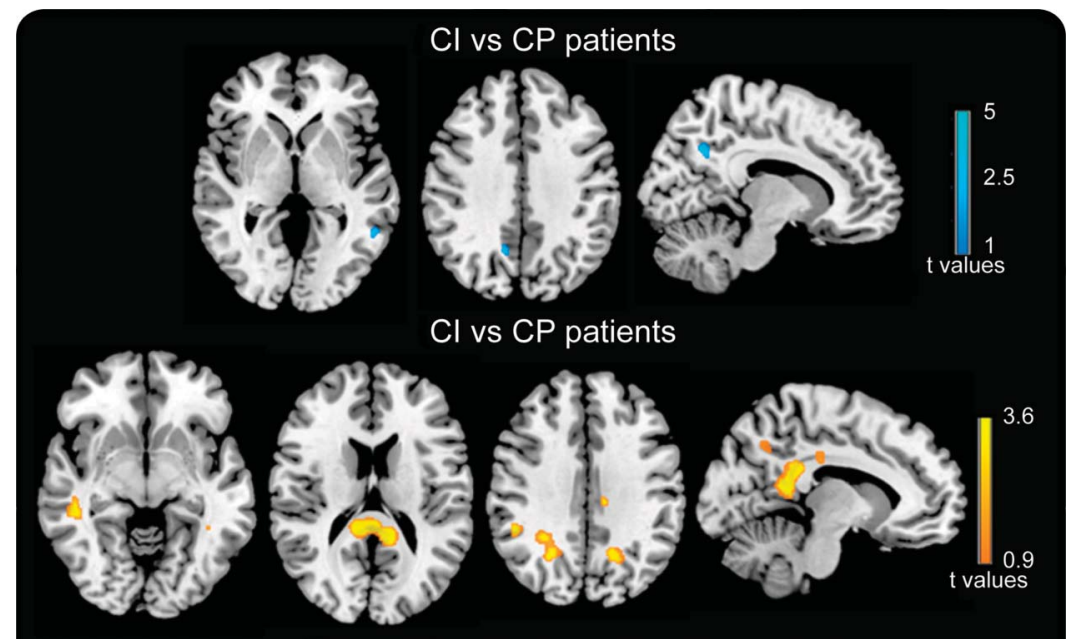

$\mathrm{Cl}$ vs $\mathrm{CP}$ patients and healthy controls

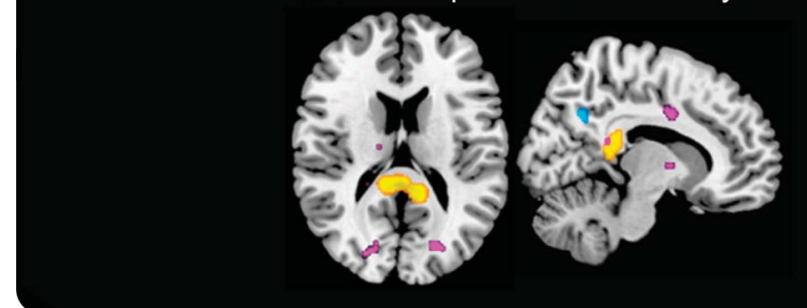

Brain regions show significant gray matter (GM) (cyan color scale) (top row) and white matter (WM) (yellow color scale) (middle row) atrophy and higher frequency of T2 lesions (pink) (bottom row) in cognitively impaired (Cl) vs cognitively preserved (CP) patients with pediatric multiple sclerosis, superimposed on a high-resolution T1-weighted template $(p<0.001$ uncorrected, cluster extent $>5$ voxels). In the bottom row, the results of the conjunction analysis showing atrophied $\mathrm{GM}$ and $\mathrm{WM}$ areas in $\mathrm{Cl}$ vs $\mathrm{CP}$ and healthy controls are shown. Images are presented in radiologic convention.

of the splenium of the CC, posterior cingulum, and WM close to the $\mathrm{L}$ precuneus (figure 1).

TBSS analysis. Compared to HC, pediatric patients with MS had a distributed pattern of decreased FA in the WM, as well as an increased RD of the splenium of the CC and posterior parieto-occipital WM. No MD or axial diffusivity abnormalities were detected (figure 2). When the 2 groups of pediatric patients with MS were analyzed separately, no DT-MRI abnormalities were detected in CP patients vs HC, while cognitively impaired patients had a diffuse pattern of decreased FA and increased $\mathrm{RD}$ mainly involving the $\mathrm{CC}$, cingulum, fornix, and parieto-occipital WM (figure 2). Compared to $\mathrm{CP}$ patients, cognitively impaired patients had decreased $\mathrm{FA}$ and increased RD of the posterior $\mathrm{CC}$ and cingulum as well as decreased FA of the bilateral parieto-occipital WM (figure 2).

DMN FC analysis. Figure 3 shows the independent component with the highest squared spatial correlation $\left(R^{2}=0.34\right.$, corresponding to a plain correlation coefficient of $r=0.58$ ) with the DMN template.
Compared to HC, pediatric patients with MS had decreased FC of the posterior regions of the DMN (R precuneus, MNI coordinates: 24, -69 , $33 ; t=3.76, k=16$; and $\mathrm{R}$ angular gyrus, $\mathrm{MNI}$ coordinates: $42,-58,42 ; t=4.76, k=24)$. CP patients vs $\mathrm{HC}$ had decreased RS FC of the R angular gyrus (MNI coordinates: 42, $-58,38 ; t=4.7, k=$ 11 , whereas cognitively impaired patients vs both HC and CP patients had decreased RS FC of the R precuneus (MNI coordinates: 24, $-67,38 ; t=3$, $k=13)$. Compared to both $\mathrm{HC}$ and cognitively impaired patients, CP patients experienced an increased RS FC of the $\mathrm{R}$ anterior cingulate cortex (ACC) (MNI coordinates: 3, 11, 46; $t=3.3, k=5$ ) (figure 3). No clusters of significantly increased RS FC were found in cognitively impaired vs $\mathrm{CP}$ patients or HC.

Analysis of correlations. Table 2 summarizes correlations ( $p<0.001$, uncorrected) between measures of regional structural and functional damage in pediatric patients with MS vs clinical, neuropsychological, and other MRI variables.

Significant correlations were found between the patterns of regional atrophy vs T2 LV, T1 LV, and global neuropsychological performance, as well as performance in individual neuropsychological tests.

Significant correlations were also found between abnormalities of RS FC vs global neuropsychological performance as well as performance on individual neuropsychological tests.

No correlation was found between regional structural or functional abnormalities and either disease duration or EDSS.

Multimodal analysis. The multivariable model identified cingulum FA, CC MD, CC RD, and R precuneus RS FC (in this order of ranking) as covariates associated with cognitive impairment $(\mathrm{C}$ index $=0.99)$.

DISCUSSION In line with previous reports, ${ }^{7,17}$ which applied similar neuropsychological tests and criteria to classify patients' cognitive status, we found that a relatively high percentage of pediatric patients with MS experience cognitive impairment, with a prominent involvement of spatial and verbal memory abilities, language, attention, and concentration. All of this indicates that our sample is representative of the more general population of patients with pediatric MS.

Using voxel-wise methods, we determined the relationship between the regional distribution of damage to the WM and GM and cognitive impairment in these patients. This analysis showed that the presence and severity of cognitive impairment was associated with structural damage to a set of brain regions that form the posterior node of the DMN. These regions had a high probability of harboring 

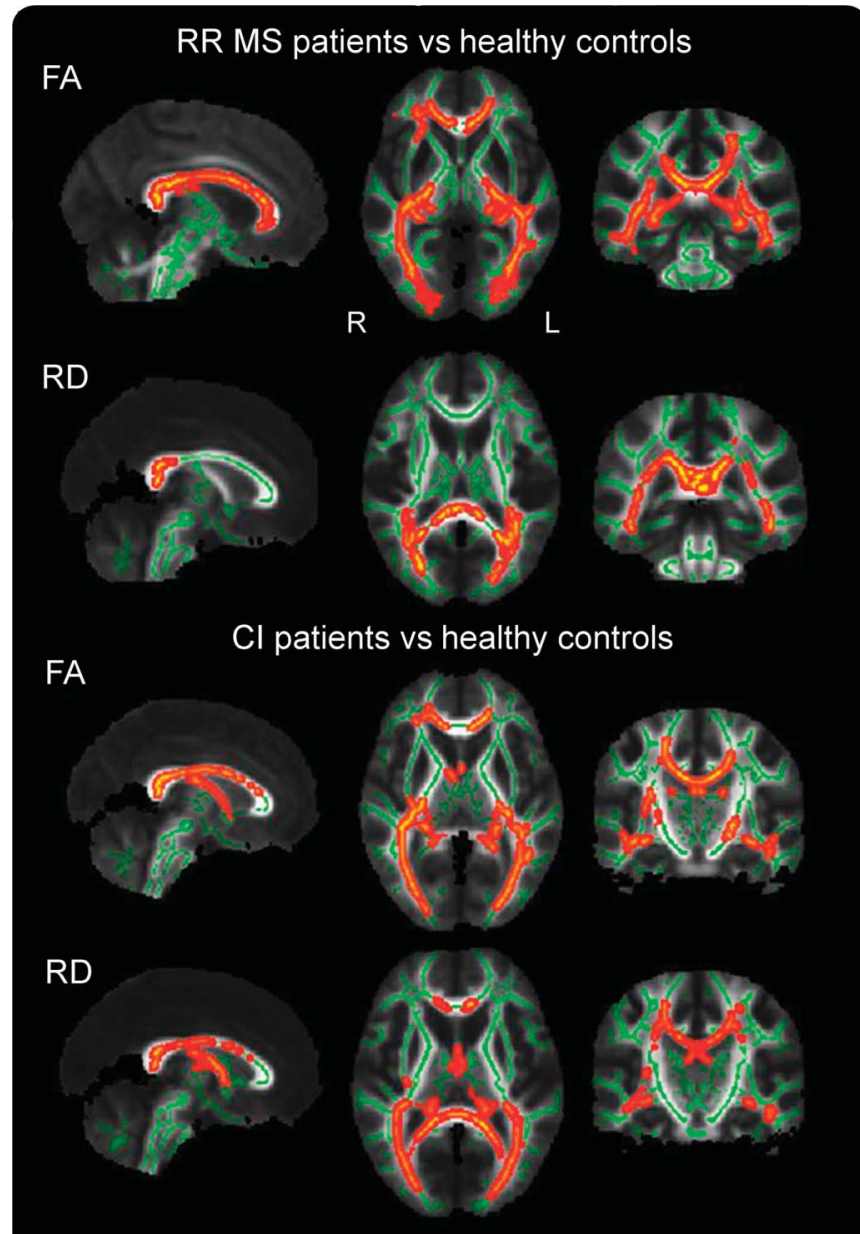

$\mathrm{Cl}$ vs $\mathrm{CP}$ patients
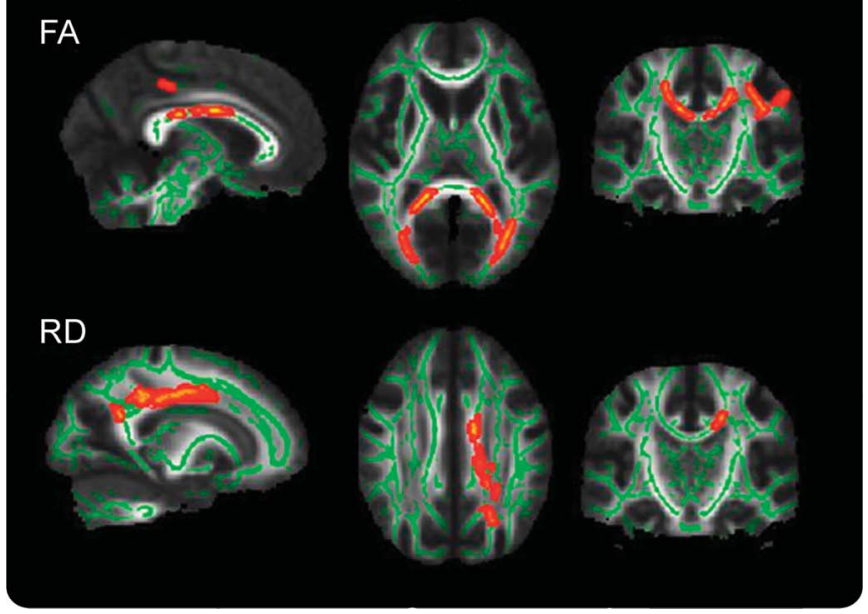

Illustrative images show in red-yellow the clusters of voxels with significantly decreased fractional anisotropy (FA) and increased radial diffusivity (RD) values among the different study groups ( $p<$ 0.01 , family-wise error corrected). The significant regions have been thickened for better visibility The white matter skeleton, thresholded at FA $>0.2$, is represented in green. The background image is the mean FA map derived from all subjects. Images are presented in radiologic convention. $\mathrm{Cl}=$ cognitively impaired; $\mathrm{CP}=$ cognitively preserved; RRMS = relapsing-remitting multiple sclerosis.

focal T2 lesions, loss of WM and GM, as well as FA and RD abnormalities. The pattern of structural abnormalities associated with cognitive impairment in pediatric patients with RRMS differs significantly from that described in adult patients with RRMS, in whom GM loss from the frontal, parietal, and temporal lobes, only marginally linked to the presence of focal lesions, has been reported consistently. ${ }^{25,26} \mathrm{~A}$ distributed pattern of WM FA abnormalities has also been detected in adult patients with MS with cognitive impairent. ${ }^{27}$ The colocalization of T2 lesions, GM and WM atrophy, and DT MRI abnormalities in brain posterior regions of pediatric patients with MS suggests that degeneration of axons passing through focal lesions and areas of demyelination (as indicated by decreased FA and increased $\mathrm{RD})^{28}$ may lead to deafferentation and atrophy, pointing to a pivotal role of WM damage (lesions and microstructural abnormalities) in the pathogenesis of cognitive impairment in these patients. This hypothesis is also supported by the results of the RF analysis, which identified FA decrease of the cingulum as the best predictor of cognitive impairment. In contrast to what has been found in adult patients with MS, ${ }^{29}$ focal GM lesions are likely not to have influenced our results, since they are found in less than $10 \%$ of pediatric patients with MS. ${ }^{5}$

In agreement with previous studies, ${ }^{30,31}$ the analysis of regional GM atrophy confirmed that thalamic atrophy is typically seen in pediatric MS and that it is strongly correlated with the extent of WM lesions, but not with disease duration. ${ }^{30,31}$ GM atrophy in our patients was not limited to the thalamus, but extended diffusely to several cortical and subcortical regions. Cognitive impairment in these patients was associated with more pronounced atrophy of the $\mathrm{R}$ precuneus and L MTG. Neuroimaging findings in healthy subjects suggest a central role of the precuneus in a wide spectrum of highly integrated tasks, including visuospatial imagery, episodic memory, and self-processing operations. ${ }^{32}$ Recently, global cognitive dysfunction has been associated with reduced thalamic volume in pediatric patients with $\mathrm{MS},{ }^{8}$ whereas word list learning has been correlated with hippocampal volume. ${ }^{33}$ Differences in the analysis methods, as well as in clinical and neuropsychological characteristics of the patients enrolled in these studies, may help to explain discrepancies between our results and previous studies. ${ }^{8,33}$ Surprisingly, patients' global cognitive performance as well as their performance on executive tests were not associated with atrophy of frontal lobe regions. Different features of cognitive impairment in adult and pediatric patients (with prominent effects on linguistic abilities in children $)^{7,17}$ as well as a different regional vulnerability to damage due to the variability of maturation and myelination of CNS structures might account for the discrepancies between pediatric and adult patients with MS. Indeed, age-related structural changes in GM and WM volumes and diffusivity characteristics, 
Figure 3 Default mode network abnormalities in pediatric multiple sclerosis
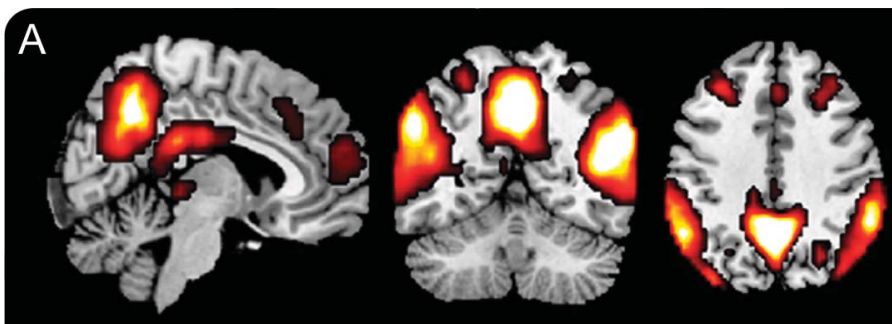

50

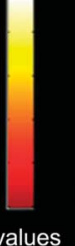

B

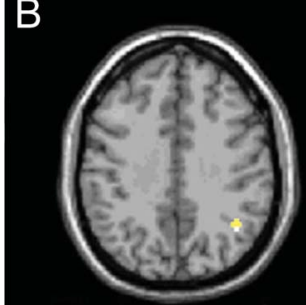

CP patients vs controls

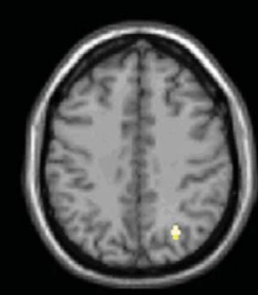

$\mathrm{Cl}$ vs $\mathrm{CP}$ patients \& controls

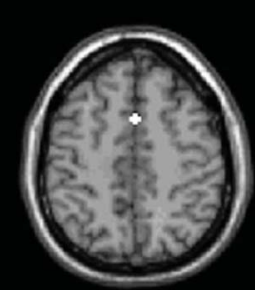

$\mathrm{CP}$ vs $\mathrm{Cl}$ patients \& controls

Spatial pattern of the independent component shows the highest squared spatial correlation with the template of the default mode network (DMN) (A). Spatial pattern of the within-group differences of resting-state (RS) functional connectivity (FC) of the DMN among the study groups (B): (1) decreased RS FC of the right angular gyrus in cognitively preserved (CP) patients with multiple sclerosis (MS) vs healthy controls; (2) decreased RS FC of the right precuneus in cognitively impaired (Cl) patients with MS vs both healthy controls and CP patients; (3) increased RS FC in the anterior cingulum in $\mathrm{CP}$ patients vs healthy controls and $\mathrm{Cl}$ patients.

with a caudo-rostral pattern of myelination, have been demonstrated consistently through childhood and adolescence by several studies. ${ }^{34,35}$ Maturation of the frontal lobe WM during the second decade of life has been suggested as a possible mechanism that confers a sort of protection from MS-related damage. ${ }^{10}$ The notion of a higher susceptibility to damage in specific brain regions of pediatric patients with MS is also supported by the analysis of the regional distribution of damage to the WM, which showed atrophy and diffusivity abnormalities in the posterior CC, cingulum, and parieto-occipital WM in cognitively impaired patients.

The analysis of RS FC of the DMN disclosed that functional abnormalities of the posterior regions of the network (in particular the precuneus) paralleled abnormalities detected by structural MRI in pediatric patients with cognitive impairment. On the other hand, CP patients experienced an increased RS FC of the ACC. The DMN is a medial cortical network involving several brain regions structurally connected throughout 2 large intrahemispheric and interhemispheric WM tracts (the CC and cingulum). ${ }^{36,37}$ Also, for the analysis of RS FC, the pattern of abnormalities we found in pediatric patients with MS with cognitive impairment differs from that described in adult patients, in whom a consistent reduced RS FC of the anterior regions of the $\mathrm{DMN}^{13,15}$ and an enhanced RS FC of the posterior ones have been described. ${ }^{13,38}$ As previously argued for the regional distribution of structural abnormalities, maturation effects might influence a different functional reorganization in adult vs pediatric patients with MS. Indeed, the long-range connections between the posterior cingulate cortex and the anterior prefrontal cortex have been shown to mature with age (being immature in 7-year-old children), ${ }^{39,40}$ and to be associated with the development of cognitive abilities. ${ }^{34}$ Importantly, the multimodal prediction model that integrated structural and functional MRI measures showed that cognitive impairment was highly associated with the extent of structural damage and reduced RS FC of the posterior node of the DMN. Localized structural damage to this node was the main factor associated with cognitive impairment. Therefore, it is tempting to speculate that abnormalities of RS FC might be secondary to the occurrence of WM damage in the same region of pediatric patients with MS.

Our study is not without limitations. First, it is cross-sectional and therefore does not allow testing for cause-effect relationships between structural and functional abnormalities. Second, the number of patients enrolled is relatively small, particularly when considering the 2 groups of patients separately. Third,

Table 2 Correlations ( $r$ values) between measures of regional structural and functional damage in pediatric patients with multiple sclerosis vs clinical, neuropsychological, and other MRI variables ( $p<0.001$ uncorrected)

$\begin{array}{llllll} & \text { T2 LV } & \text { T1 LV } & \begin{array}{l}\text { Number of abnormal } \\ \text { neuropsychological tests }\end{array} & \text { TMT-B } & \text { SRT-LTS } \\ \text { Left thalamus volume } & -0.65 & -0.70 & - & - & - \\ \text { SPART-D }\end{array}$

Abbreviations: $C C=$ corpus callosum; LV = lesion volume; SPART = 10/36 Spatial Recall Test; SPART-D = 10/36 Spatial Recall Test-Delayed; SRT-LTS = Selective Reminding Test-Long-Term Storage; TMT = Trail Making Test. 
disease duration of the cohort was relatively short and their EDSS score relatively low, thus potentially influencing our correlation analyses. Finally, we did not perform a neuropsychological evaluation (including assessment of global IQ) in controls.

\section{AUTHOR CONTRIBUTIONS}

Maria A. Rocca contributed to drafting/revising the manuscript, statistical analysis, and obtaining funding. Martina Absinta contributed to MRI data postprocessing and statistical analysis. Maria Pia Amato contributed to patients' enrollment and analysis of the data. Lucia Moiola contributed to patients' enrollment and analysis of the data. Angelo Ghezzi contributed to patients' enrollment and analysis of the data. Pierangelo Veggiotti contributed to patients' enrollment and analysis of the data. Ruggero Capra contributed to patients' enrollment and analysis of the data. Emilio Portaccio contributed to patients' neuropsychological assessment and analysis of the data. Agnese Fiorino contributed to patients' neuropsychological assessment and analysis of the data. Lorena Pippolo contributed to patients' neuropsychological assessment and analysis of the data. Maria Carmela Pera contributed to patients' neuropsychological assessment and analysis of the data. Mark A. Horsfield contributed to drafting/ revising the manuscript and interpretation of the data. Andrea Falini contributed to MRI acquisitions and analysis of the data. Giancarlo Comi contributed to the study concept. Massimo Filippi contributed to drafting/revising the manuscript, study concept, and analysis and interpretation of the data. He also acted as study supervisor. All authors approved the current version of the manuscript.

\section{STUDY FUNDING}

Supported by a grant from the Italian Ministry of Health (GR-20091529671).

\section{DISCLOSURE}

M. Rocca received speakers honoraria from Biogen Idec and Serono Symposia International Foundation and receives research support from the Italian Ministry of Health. M. Absinta reports no disclosures relevant to the manuscript. M. Amato serves on scientific advisory boards for Biogen-Idec, Merck Serono, Bayer Schering, and Sanofi-Aventis and receives research support and honoraria for speaking from Biogen-Idec, Merck Serono, Bayer Schering, and Sanofi-Aventis. L. Moiola reports no disclosures relevant to the manuscript. A. Ghezzi has served on scientific advisory boards for Merck Serono, Biogen Idec, and Teva Pharmaceutical Industries Ltd.; has received speaker honoraria from Merck Serono, Biogen Idec, Bayer Schering Pharma, Novartis, and Serono Symposia International; served as a consultant for Novartis; and receives research support from Sanofi-Aventis, Biogen Idec, and Merck Serono. P. Veggiotti reports no disclosures relevant to the manuscript. R. Capra received consulting fees from Novartis, Merck Serono, and Biogen Idec and lecture fees from Bayer, BiogenIdec, Dompé, Genzyme, and Sanofi-Aventis. E. Portaccio serves on a scientific advisory board for Biogen-Idec, Merck Serono, and Bayer, received honoraria for speaking from Biogen-Idec and Teva, and receives research support from Merck Serono, Biogen-Idec, Bayer Schering, and Sanofi-Aventis. A. Fiorino, L. Pippolo, and M. Pera report no disclosures relevant to the manuscript. M. Horsfield has acted as a consultant to Biogen Idec and to GE Healthcare, and is a stockholder of Xinapse Systems. A. Falini reports no disclosures relevant to the manuscript. G. Comi has received consulting fees for participating on advisory boards from Novartis, Teva Pharmaceutical Ind. Ltd., Sanofi, Genzyme, Merck Serono, Bayer, and Actelion, and honorarium for speaking activities for Novartis, Teva Pharmaceutical Ind. Ltd., Sanofi, Genzyme, Merck Serono, Bayer, Biogen, and Serono Symposia International Foundation. M. Filippi serves on scientific advisory boards for Teva Pharmaceutical Industries Ltd. and Genmab A/S; has received funding for travel from Bayer Schering Pharma, Biogen Idec, Genmab A/S, Merck Serono, and Teva Pharmaceutical Industries Ltd.; serves as a consultant to Bayer Schering Pharma, Biogen Idec, Genmab A/S, Merck Serono, Novartis, Pepgen Corporation, and Teva Pharmaceutical Industries Ltd.; serves on speakers' bureaus for Bayer Schering Pharma, Biogen Idec, Genmab A/S, Merck Serono, and Teva
Pharmaceutical Industries Ltd.; and receives research support from Bayer Schering Pharma, Biogen Idec, Genmab A/S, Novartis, Merck Serono, Teva Pharmaceutical Industries Ltd., Fondazione Italiana Sclerosi Multipla, the Italian Ministry of Health, and CurePSP. Go to Neurology.org for full disclosures.

Received July 3, 2013. Accepted in final form November 18, 2013.

\section{REFERENCES}

1. Degenhardt A, Ramagopalan SV, Scalfari A, Ebers GC. Clinical prognostic factors in multiple sclerosis: a natural history review. Nat Rev Neurol 2009;5:672-682.

2. Renoux C, Vukusic S, Mikaeloff Y, et al. Natural history of multiple sclerosis with childhood onset. N Engl J Med 2007;356:2603-2613.

3. Tortorella P, Rocca MA, Mezzapesa DM, et al. MRI quantification of gray and white matter damage in patients with early-onset multiple sclerosis. J Neurol 2006;253: 903-907.

4. Absinta M, Rocca MA, Moiola L, et al. Brain macro-and microscopic damage in patients with paediatric MS. J Neurol Neurosurg Psychiatry 2010;81:1357-1362.

5. Absinta M, Rocca MA, Moiola L, et al. Cortical lesions in children with multiple sclerosis. Neurology 2011;76: 910-913.

6. Rocca MA, Absinta M, Ghezzi A, Moiola L, Comi G, Filippi M. Is a preserved functional reserve a mechanism limiting clinical impairment in pediatric MS patients? Hum Brain Mapp 2009;30:2844-2851.

7. Amato MP, Goretti B, Ghezzi A, et al. Cognitive and psychosocial features of childhood and juvenile MS. Neurology 2008;70:1891-1897.

8. Till C, Ghassemi R, Aubert-Broche B, et al. MRI correlates of cognitive impairment in childhood-onset multiple sclerosis. Neuropsychology 2011;25:319-332.

9. Till C, Ho C, Dudani A, Garcia-Lorenzo D, Collins DL, Banwell BL. Magnetic resonance imaging predictors of executive functioning in patients with pediatric-onset multiple sclerosis. Arch Clin Neuropsychol 2012;27:495-509.

10. Bethune A, Tipu V, Sled JG, et al. Diffusion tensor imaging and cognitive speed in children with multiple sclerosis. J Neurol Sci 2011;309:68-74.

11. Till C, Deotto A, Tipu V, et al. White matter integrity and math performance in pediatric multiple sclerosis: a diffusion tensor imaging study. Neuroreport 2011;22:1005-1009.

12. Damoiseaux JS, Beckmann CF, Arigita EJ, et al. Reduced resting-state brain activity in the "default network" in normal aging. Cereb Cortex 2008;18:1856-1864.

13. Bonavita S, Gallo A, Sacco R, et al. Distributed changes in default-mode resting-state connectivity in multiple sclerosis. Mult Scler 2011;17:411-422.

14. Hawellek DJ, Hipp JF, Lewis CM, Corbetta M, Engel AK. Increased functional connectivity indicates the severity of cognitive impairment in multiple sclerosis. Proc Natl Acad Sci USA 2011;108:19066-19071.

15. Rocca MA, Valsasina P, Absinta M, et al. Default-mode network dysfunction and cognitive impairment in progressive MS. Neurology 2010;74:1252-1259.

16. Krupp LB, Banwell B, Tenembaum S. Consensus definitions proposed for pediatric multiple sclerosis and related disorders. Neurology 2007;68:S7-S12.

17. Portaccio E, Goretti B, Lori S, et al. The brief neuropsychological battery for children: a screening tool for cognitive impairment in childhood and juvenile multiple sclerosis. Mult Scler 2009;15:620-626. 
18. Smith SM, Jenkinson M, Johansen-Berg H, et al. Tractbased spatial statistics: voxelwise analysis of multi-subject diffusion data. Neuroimage 2006;31:1487-1505.

19. Nichols TE, Holmes AP. Nonparametric permutation tests for functional neuroimaging: a primer with examples. Hum Brain Mapp 2002;15:1-25.

20. Calhoun VD, Adali T, Pearlson GD, Pekar JJ. A method for making group inferences from functional MRI data using independent component analysis. Hum Brain Mapp 2001;14:140-151.

21. Franco AR, Pritchard A, Calhoun VD, Mayer AR. Interrater and intermethod reliability of default mode network selection. Hum Brain Mapp 2009;30:2293-2303.

22. Brett M, Anton JL, Valabregue R, Poline JP. Region of interest analysis using an SPM toolbox. Neuroimage 2002; 16:372.

23. Breiman L. Random forests. Mach Learn 2001;45:5-32.

24. Wechsler D. WISC-R Manual for the Wechsler Intelligence Scale for Children-Revised. New York: The Psychological Corporation; 1974.

25. Morgen K, Sammer G, Courtney SM, et al. Evidence for a direct association between cortical atrophy and cognitive impairment in relapsing-remitting MS. Neuroimage 2006; 30:891-898.

26. Riccitelli G, Rocca MA, Pagani E, et al. Cognitive impairment in multiple sclerosis is associated to different patterns of gray matter atrophy according to clinical phenotype. Hum Brain Mapp 2011;32:1535-1543.

27. Hulst HE, Steenwijk MD, Versteeg A, et al. Cognitive impairment in MS: impact of white matter integrity, gray matter volume, and lesions. Neurology 2013;80:1025-1032.

28. Wheeler-Kingshott CA, Cercignani M. About "axial" and "radial" diffusivities. Magn Reson Med 2009;61: 1255-1260.

29. Calabrese M, Agosta F, Rinaldi F, et al. Cortical lesions and atrophy associated with cognitive impairment in relapsing-remitting multiple sclerosis. Arch Neurol 2009; 66:1144-1150.
30. Mesaros S, Rocca MA, Absinta M, et al. Evidence of thalamic gray matter loss in pediatric multiple sclerosis. Neurology 2008;70:1107-1112.

31. Aubert-Broche B, Fonov V, Ghassemi R, et al. Regional brain atrophy in children with multiple sclerosis. Neuroimage 2011;58:409-415.

32. Cavanna AE, Trimble MR. The precuneus: a review of its functional anatomy and behavioural correlates. Brain 2006;129:564-583.

33. Fuentes A, Collins DL, Garcia-Lorenzo D, et al. Memory performance and normalized regional brain volumes in patients with pediatric-onset multiple sclerosis. J Int Neuropsychol Soc 2012;18:471-480.

34. Paus T. Mapping brain maturation and cognitive development during adolescence. Trends Cogn Sci 2005;9: 60-68.

35. Klingberg T, Vaidya CJ, Gabrieli JD, Moseley ME, Hedehus M. Myelination and organization of the frontal white matter in children: a diffusion tensor MRI study. Neuroreport 1999;10:2817-2821.

36. van den Heuvel M, Mandl R, Luigjes J, Hulshoff Pol H. Microstructural organization of the cingulum tract and the level of default mode functional connectivity. J Neurosci 2008;28:10844-10851.

37. Greicius MD, Supekar K, Menon V, Dougherty RF. Restingstate functional connectivity reflects structural connectivity in the default mode network. Cereb Cortex 2009;19:72-78.

38. Roosendaal SD, Schoonheim MM, Hulst HE, et al. Resting state networks change in clinically isolated syndrome. Brain 2010;133:1612-1621.

39. Fair DA, Dosenbach NU, Church JA, et al. Development of distinct control networks through segregation and integration. Proc Natl Acad Sci USA 2007;104: 13507-13512.

40. Supekar K, Uddin LQ, Prater K, Amin H, Greicius MD, Menon V. Development of functional and structural connectivity within the default mode network in young children. Neuroimage 2010;52:290-301.

\section{Enjoy Big Savings on NEW 2014 AAN Practice Management Webinars Subscriptions}

The American Academy of Neurology offers 14 cost-effective Practice Management Webinars you can attend live or listen to recordings posted online. AAN members can purchase one webinar for $\$ 149$ or subscribe to the entire series for only $\$ 199$. This is new pricing for 2014 and significantly less than 2013-and big savings from the new 2014 nonmember price of $\$ 199$ per webinar or $\$ 649$ for the subscription. Register today for these and other 2014 webinars at AAN.com/view/pmw14:

April 8 - How PQRS Quality Measures Will Inform Future Medicare Value-based Payments

May 13 - Measuring and Improving Your Patients' Experience

June 18 - Using Practice Benchmarking Analytics to Improve Your Bottom Line 